\title{
Improved strategies for branching on general disjunctions
}

\author{
Gerard Cornuéjols ${ }^{1}$ Leo Liberti ${ }^{2}$ Giacomo Nannicini ${ }^{2}$
}

Key words: Integer programming, branch and bound, split disjunctions

\section{Extended abstract}

In this paper we consider the Mixed Integer Linear Program in standard form:

$$
\left.\begin{array}{c}
\min c^{\top} x \\
A x=b \\
x \geq 0 \\
\forall j \in N_{I} \quad x_{j} \in \mathbb{Z},
\end{array}\right\} \mathcal{P}
$$

where $c \in \mathbb{R}^{n}, b \in \mathbb{R}^{m}, A \in \mathbb{R}^{m \times n}$ and $N_{I} \subset N=\{1, \ldots, n\}$. The LP relaxation of (1) is the linear program obtained by dropping the integrality constraints, and is denoted by $\overline{\mathcal{P}}$. The Branch-and-Bound algorithm makes an implicit use of the concept of disjunctions [1]: whenever the solution of the current relaxation is fractional, we divide the current problem $\mathcal{P}$ into two subproblems $\mathcal{P}_{1}$ and $\mathcal{P}_{2}$ such that the union of the feasible regions of $\mathcal{P}_{1}$ and $\mathcal{P}_{2}$ contains all feasible solutions to $\mathcal{P}$. Usually, this is done by choosing a fractional component $\bar{x}_{i}$ (for some $i \in N_{I}$ ) of the optimal solution $\bar{x}$ to the relaxation $\overline{\mathcal{P}}$, and adding the constraints $x_{i} \leq\left\lfloor\bar{x}_{i}\right\rfloor$ and $x_{i} \geq\left\lceil\bar{x}_{i}\right\rceil$ to $\mathcal{P}_{1}$ and $\mathcal{P}_{2}$ respectively.

Email addresses: gc0v@andrew.cmu.edu (Gerard Cornuéjols), liberti@lix.polytechnique.fr (Leo Liberti), giacomon@lix.polytechnique.fr (Giacomo Nannicini).

1 LIF, Faculté de Sciences de Luminy, Marseille, France and Tepper School of Businness, Carnegie Mellon University, Pittsburgh, PA

2 LIX, École Polytechnique, 91128 Palaiseau, France 
Within this paper, we take the more general approach whereby branching can occur with respect to a direction $\pi \in \mathbb{R}^{n}$ by adding the constraints $\pi x \leq \beta_{0}$, $\pi x \geq \beta_{1}$ with $\beta_{0}<\beta_{1}$ to $\mathcal{P}_{1}$ and $\mathcal{P}_{2}$ respectively, as long as no integer feasible point is cut off. Karamanov and Cornuéjols [2] proposed using disjunctions arising from Gomory Mixed-Integer Cuts generated directly from the rows of the optimal tableau. We consider split disjunctions arising from Gomory Mixed-Integer Cuts generated from linear combinations of the rows of the simplex tableau; by generating combination that yield a stronger intersection cut, we generate split disjunctions that cut deeply into the feasible region, thereby reducing the total number of nodes in the enumeration tree. By combining branching on simple disjunctions and on general disjunctions we obtain an improvement over traditional branching rules on the majority of test instances.

\section{References}

[1] E. Balas. Disjunctive programming. Annals of Discrete Mathematics, 5:3-51, 1979.

[2] M. Karamanov and G. Cornuéjols. Branching on general disjunctions. Technical report, Carnegie Mellon University, 2005. 